\title{
miR-19a-3p Functions as an Oncogene by Regulating FBXO32 Expression in Multiple Myeloma
}

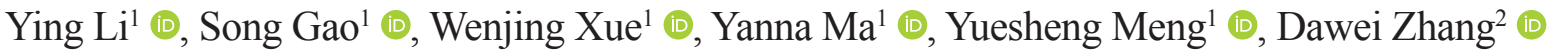

${ }^{1}$ Department of Hematology, Jinshan Hospital of Fudan University, Jinshan, Shanghai, PR China

${ }^{2}$ Department of General Surgery, Jinshan Hospital of Fudan University, Jinshan, Shanghai, PR China

Background: Multiple myeloma remains a virtually incurable hematologic malignancy, which is featured with the aberrant growth of malignant plasma cells.

Aims: To elucidate the functions of miR-19a-3p in multiple myeloma. Study Design: Cell study.

Methods: Cell counting kit- 8 assay was performed to detect cell viability, and flow cytometry was conducted to detect cell apoptosis. Bioinformatics analysis predicted miR-19a-3p-associated biological function, pathway, core regulatory network, and target genes. Luciferase reporter assay verified the target sequence of miR-19a-3p regulating FBXO32.
Results: miR-19a-3p is upregulated in multiple myeloma cells $(\mathrm{p}<0.01)$ and patients with multiple myeloma $(\mathrm{p}<0.001)$. Overexpressed miR$19 a-3 p$ significantly increased cell viability $(p<0.05)$ and inhibited cell apoptosis $(p<0.01)$. FBXO32 is a target gene of miR-19a-3p $(p<0.01)$. Moreover, FBXO32 is downregulated in MM, and it significantly decreased cell viability $(p<0.05)$ and promoted cell apoptosis $(p<0.01)$. FBXO32 significantly rescued the influence of miR-19a-3p-inhibiting cell apoptosis $(\mathrm{p}<0.05)$.

Conclusion: miR-19a-3p promoted cell proliferation and inhibited cell apoptosis by degrading the target FBXO32 mRNA in multiple myeloma.
Multiple myeloma (MM), accounting for approximately $13 \%$ of hematological malignancies, is featured with the aberrant growth of malignant plasma cells (1). Previous research achievements have greatly increased life expectancy and quality of life. However, MM remains a virtually incurable hematologic malignancy (2). MM pathogenesis is involved in a series of biological processes, such as gene mutations, chromosomal abnormalities, epigenetic modifications, cell proliferation, tumor-microenvironment, and evolution of drug-resistant tumor cells $(3,4)$. Identifying essential genes in disease progression is of the most significant importance for identifying new therapeutic approaches and prolonging the prognosis in patients $(5,6)$.

MicroRNAs (miRNAs), 18-24 nucleotides in length, are a kind of noncoding RNA. miRNAs regulate posttranscription gene expression by degrading or repressing target mRNAs $(7,8)$. miRNAs mediate various biological functions such as differentiation, proliferation, apoptosis, and migration $(9,10)$. A series of cancer-associated miRNAs have recently been profiled in MM using microarray analysis (10). Previous findings demonstrated that miR-19a-3p acts as a novel poor prognostic indicator in MM (11). However, the mechanism of miR-19a-3p regulating cellular function in myeloma cells has not been elucidated.
FBXO32 (atrogin-1) belongs to the F-box protein family (12). Recent findings have reported that FBXO32 is down-regulated in cancers and may function as a tumor suppressor (13-17). Besides, emerging studies suggest that FBXO32 is a novel apoptosis regulator (18). However, the upstream regulatory factor of FBXO32 in tumorigenesis remains unclear.

This study showed that miR-19a-3p is significantly overexpressed and significantly suppressed cell apoptosis in MM cells. FBXO32 was negatively regulated by miR-19a-3p. Besides, our results demonstrated that FBXO32 was a miR-19a-3p target gene in MM cells. FBXO32 rescued the function of miR-19a-3p inhibiting cell apoptosis in MM cells. These results suggested that miR-19a-3p promoted MM cells development by regulating FBXO32 expression.

\section{MATERIALS AND METHODS}

Cell lines and clinical specimens

Cells (MM.1S, U266, RPMI8266, IM9, H929, and W63) were purchased from the American Type Culture Collection (MD, USA). Bone marrow (BM) specimens were provided by 8 healthy donors ( 5 males, 3 females; age range $=25-55$ years) and 8 patients with MM (4 males, 4 females; age range $=30-56$ years) from (for blind review). Clinical samples were purified with CD138 MicroBeads

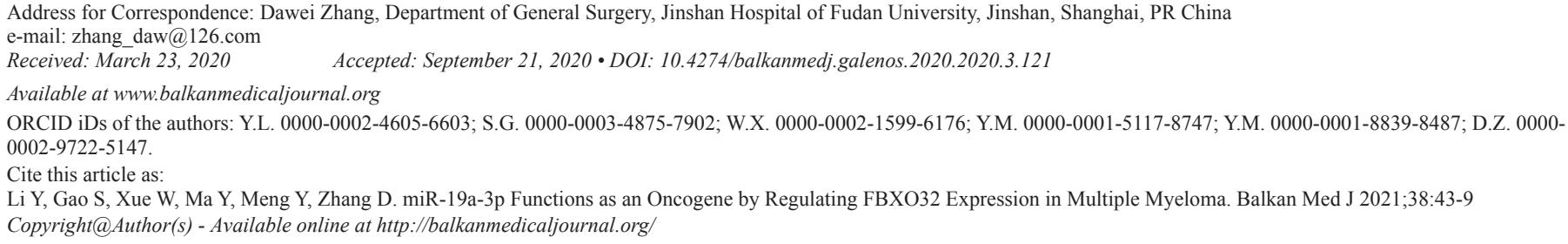


according to the Miltenyi Biotec protocol. Ethical approval was obtained from Jinshan Hospital of Fudan University. Cells were grown in the Roswell Park Memorial Institute 1640 medium, containing 10\% fetal bovine serum, penicillin $(100 \mathrm{U} / \mathrm{mL})$, and streptomycin $(100 \mu \mathrm{g} / \mathrm{mL})$.

\section{Quantitative real-time polymerase chain reaction}

We extracted total RNAs (cells and BM specimens) using TRizol reagent (Invitrogen, MD, USA) following the manufacturer's instructions. The SYBR Green primers are listed in Table 1. Reverse transcription of complementary DNA and quantitative real-time

TABLE 1. The primer sequences

\begin{tabular}{ll}
\hline Gene & Sequence $\left(5^{\prime} \rightarrow 3\right.$ ') \\
\hline miR-19a-3p-forward primer & GGGGGGGTGTGCAAATCT \\
miR-19a-3p-reverse primer & GTGCGTGTCGTGGAGTCG \\
FBXO32-forward primer & GCCTTTGTGCCTACAACTGAA \\
FBXO32-reverse primer & CTGCCCTTTGTCTGACAGAAT \\
GAPDH-forward primer & GGAGCGAGATCCCTCCAAAAT \\
GAPDH-reverse primer & GGCTGTTGTCATACTTCTCATGG \\
U6-forward primer & GCTTCGGCAGCACATATACTAAAAT \\
U6-reverse primer & CGCTTCACGAATTTGCGTGTCAT \\
\hline
\end{tabular}

polymerase chain reaction (RT-qPCR) was performed using Takara reagent (Takara, Shiga, Japan). Glyceraldehyde 3-phosphate dehydrogenase (GAPDH) and U6 were conducted as the standard for normalization. The $2^{-\Delta \Delta \mathrm{CT}}$ method was used to determine mRNA expression levels.

\section{Transfection}

Invitrogen (Thermo Fisher Scientific, Waltham, MA, USA) was responsible for synthesizing the miR-19a-3p mimics (cat. no. MIMAT0000073) and inhibitors (cat. no. MIMAT0021837). FBXO32 overexpression vector was synthesized by Sangon Biotech (Shanghai, China). Vectors were transfected using lipofectamine 2000 (Invitrogen, Carlsbad, CA, USA).

\section{Cell counting Kit-8 assays}

Cell Counting Kit-8 (CCK-8) (DOJINDO, Japan) reagent was used to test cell viability. U266 and H929 cells $\left(5 \times 10^{3}\right.$ per well) were seeded on plates and incubated for 24 hours, 48 hours, or 72 hours. The medium was replaced with $10 \mu \mathrm{L} \mathrm{CCK}-8$ reagent and $100 \mu \mathrm{L}$ fresh medium. After 1 hour at $37^{\circ} \mathrm{C}$, the wavelength of optical density values was $450 \mathrm{~nm}$.

\section{Flow cytometry}

The FITC/PI reagent (KeyGen Biotech Co, Nanjing, China) was used to detect cell apoptosis. FlowJo_V10 (Becton-Dickinson, a

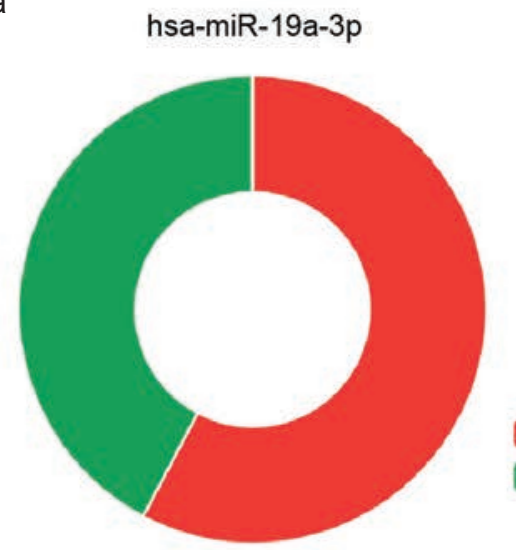

C

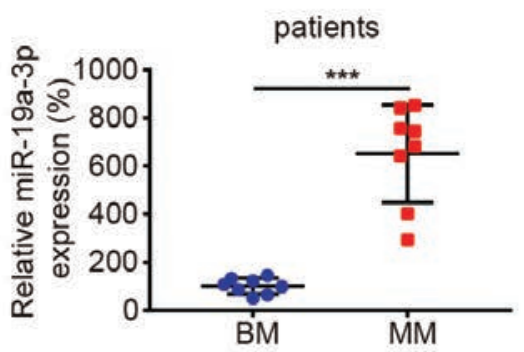

inducing angiogenesis

sustaining proliferative signaling

d

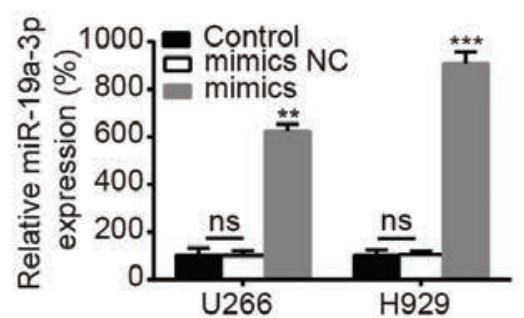

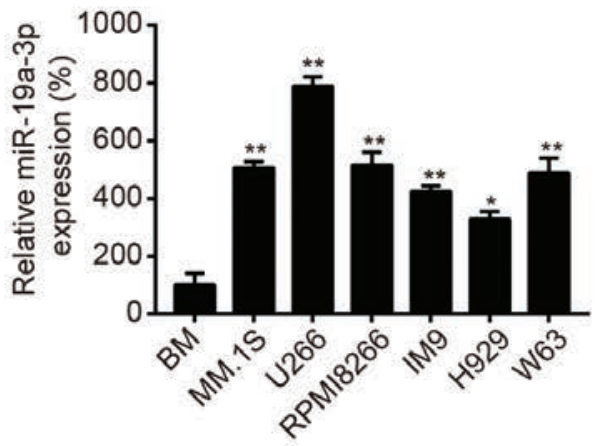

e

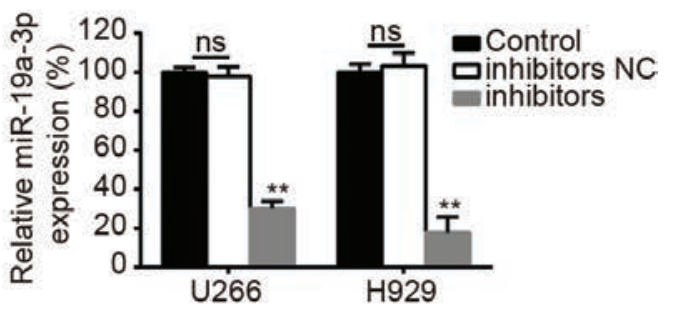

FIG. 1. a-e. Expression of miR-19a-3p in multiple myeloma (MM) cell lines and tissues. Association between miR-19a-3p and hallmarks of cancer from Cancer Hallmarks Analytics Tool (CHAT), which can be accessed at http://chat.lionproject.net/ (a). Relative miR-19a-3p expression levels between normal bone marrow (BM) cells and MM cell lines (b). Relative miR-19a-3p expression levels between normal BM cells and patients with MM (c). miR-19a-3p mimics increased miR-19a-3p expression in U266 and H929 cells (d). miR-19a-3p inhibitors suppressed miR-19a-3p expression in U266 and H929 cells (e). * $p<0.05$; ${ }^{* *} p<0.01$; ${ }^{* * *} p<0.001$; each bar represented mean \pm standard deviation from 3 independent experiments 


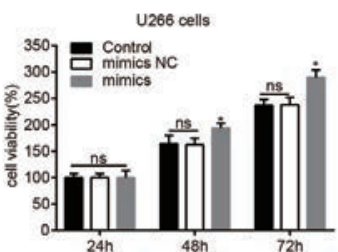

b

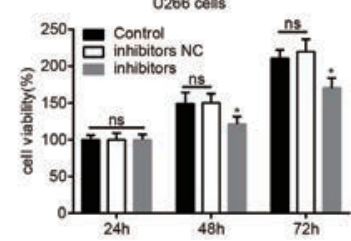

C

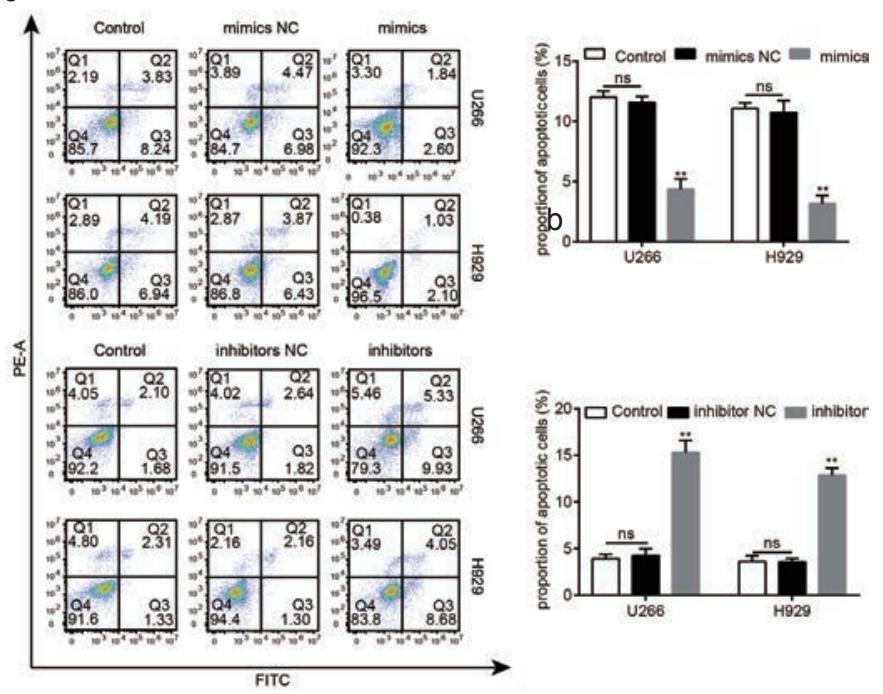

FIG. 2. a-c. miR-19a-3p promoted cell viability and inhibited cell apoptosis in multiple myeloma (MM) cell lines. miR-19a-3p overexpression promoted cell viability in U266 cells and H929 cells (a). miR-19a-3p inhibitors decreased cell viability in U266 cells and H929 cells (b). miR-19a-3p overexpression inhibited cell apoptosis, and miR-19a-3p inhibitors promoted cell apoptosis in U266 cells and H929 cells (c). ${ }^{*} p<0.05$; ${ }^{* *} p<0.01$; each bar represented mean \pm standard deviation from 3 independent experiments

New Jersey, USA) software was used to analyze the results. The upper right quadrant represented late apoptotic cells. The right lower quadrant represented viable apoptotic cell.

\section{Bioinformatics analyses}

The biological function of miR-19a-3p and FBXO32 in cancer was explored in the Cancer Hallmarks Analytics Tool (CHAT) (19). Targets of miR-19a-3p were analyzed by TargetScan (20), miRDB (21), and DIANA-microT (22) database. Online Venn tools (http:// bioinformatics.psb.ugent.be/webtools/Venn/) visualized the overlapping target genes of these databases. Enrichment analysis (gene ontology and pathway) was performed using the Enrichr analysis tool (23). The protein interaction network of miR-19a-3p target genes was analyzed using the STRING analysis tool (https://stringdb.org) (24) and visualized by Cytoscape software. The Cytoscape MCODE algorithm further extracted the core subnetwork.

\section{Dual-luciferase reporter assays}

The estimated binding site of miR-19a-3p or mutant 3'-UTR sequences were cloned in the XhoI and Notl (Promega Corporation, Madison, WI, USA) restriction sites to the psiCHECK2 vector. The cells were cotransfected with the vectors of wild-type or mutated FBXO32 3'-UTR and the miR-19a-3p NC/mimics/inhibitors. Luciferase assay reagent (Promega Corporation, Madison, WI, USA) was conducted to detect the luciferase activity after 48 hours.

\section{Western blotting}

Cleaved caspase 3 and B-cell lymphoma-2 BCL-2 (Abcam, San Francisco, CA, USA) expression levels were detected by western blotting using following the manufacturer's instructions. Cells were lysed with lysis buffer (Cell Signaling Technology, Inc, Danvers, MA, USA). The supernatant protein was collected and normalized, and equivalent protein $(30 \mu \mathrm{g})$ was electrophoresed and then transferred onto the polyvinylidene difluoride (PVDF) membranes. A blocking buffer was used to block proteins on the PVDF membranes at $4^{\circ} \mathrm{C}$ overnight. Then, the membranes incubated with the specific antibody and were washed in washing buffer $(0.1 \%$ Tween 20 in phosphate-buffered saline). Proteins were then visualized by electrochemiluminescence reagents (KeyGen Biotech Co, Nanjing, China). GAPDH (ImmunoWay Biotechnology Company, Plano, TX, USA) expression levels were used as the standard for normalization.

\section{Statistical analysis}

We analyzed the results using the Statistical Package for the Social Sciences version 21.0 software (IBM SPSS Corp, Armonk, NY, USA). Normality distribution of variables was tested using the Shapiro Wilk test. Mean \pm standard deviation was used as descriptive statistics for normal distributed data. Median (min-max) was used as descriptive statistics for non-normal distributed data. The $t$ test and 1-way analysis of variance tested normally distributed results. Multiple comparisons were made using least significant difference and Student-Newman-Keuls tests. Either $\chi^{2}$ tests or Fisher exact tests were used to analyze non-normal distributed data. All experiments presented here were conducted at least 3 independent times. $\mathrm{p}<0.05$ was considered statistically significant.

\section{RESULTS}

\section{Basic expression levels of miR-19a-3p in multiple myeloma cells and patients}

Using CHAT, we revealed that miR-19a-3p is associated with inducing angiogenesis and sustaining proliferative signaling (Figure 1a). RT-qPCR assays revealed that miR-19a-3p was increased in MM cells and patients $(\mathrm{p}<0.01)$ (Figure 1. b, c).

\section{miR-19a-3p regulated cell viability and apoptosis in multiple myeloma cells}

To further investigate the biological function of miR-19a-3p in MM, we overexpressed or knocked down miR-19a-3p in U266 and H929 cells $(\mathrm{p}<0.01)$ (Figure 1. d, e). CCK-8 assays revealed that overexpressed miR-19a-3p significantly promotes cell viability $(\mathrm{p}<0.05)$. Besides, miR-19a-3p knockdown suppressed cell viability $(\mathrm{p}<0.05)$ (Figure 2 . a, b). Flow cytometry was conducted to detect cell apoptosis in U266 and H929 cells, which showed that miR-19a-3p overexpression significantly inhibits cell apopto- 
b \begin{tabular}{l} 
GO: Biological Process \\
regulation of mesenchymal stem cell differentiation (GO:2000739) \\
negative regulation of cytoplasmic translation (GO:2000766) \\
protein K11-linked deubiquitination (GO:0035871) \\
Rap protein signal transduction (GO:0032486) \\
NLS-bearing protein import into nucleus (GO:0006607) \\
stresS-activated protein kinase signaling cascade (GO:0031098) \\
regulation of Golgi organization (GO:1903358) \\
regulation of defense response to virus (GO:0050688) \\
\hline ventricular septum morphogenesis (GO:0060412) \\
positive regulation of phosphoprotein phosphatase activity (GO:0032516)
\end{tabular} ribonucleoprotein granule (GO:0035770) trans-Golgi network transport vesicle (GO:0030140) clathrin-coated vesicle (GO:0030136) trans-Golgi network (GO:0005802) messenger ribonucleoprotein complex (GO:1990124) axon (GO:0030424) early endosome (GO:0005769) microtubule end (GO:1990752) cytoplasmic ribonucleoprotein granule (GO:0036464) node of Ranvier (GO:0033268)
C GO: Molecular Function kinase activity (GO:0004674) protein kinase activity (GO:0004672) ubiquitin-protein transferase activity (GO:0004842) ubiquitin-like protein ligase activity (GO:0061659) poly-purine tract binding (GO:0070717) ubiquitin protein ligase activity (GO:0061630) transcription factor activity, RNA polymerase II core promoter proximal MAP kinase activity (GO:0004707) region sequence-specific binding (GO:0000982) core promoter proximal region sequence-specific DNA binding (GO:0000987) thiol-dependent ubiquitin-specific protease activity (GO:0004843)

e Prolactin signaling pathway KEGG 2019 Human FoxO signaling pathway mTOR signaling pathway Rap1 signaling pathway Signaling pathways regulating pluripotency of stem cells Autophagy Cellular senescence Focal adhesion Proteoglycans in cancer GnRH signaling pathway

f

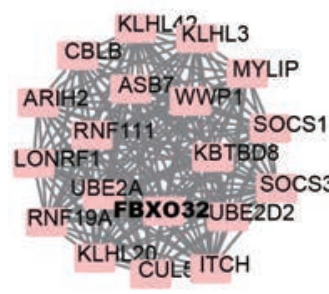

Cluster 1 (score: 19$)$

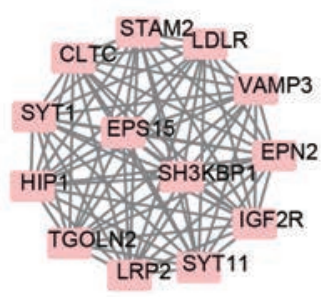

Cluster 2 (score: 13)

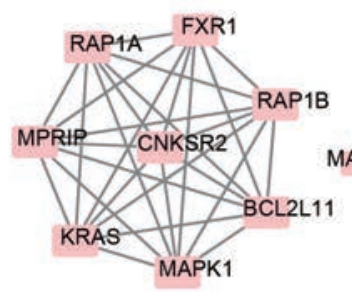

Cluster 3 (score: 8 )

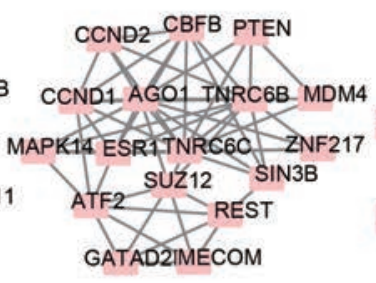

Cluster 4 (score: 7 )

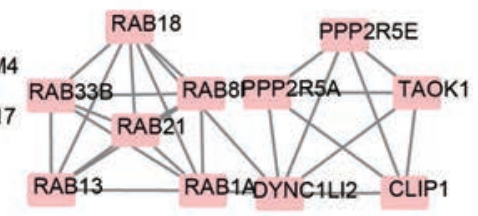

Cluster 5 (score: 5.4 )

FIG. 3. a-f. Predicting the target genes of miR-19a-3p by TargetScan, miRDB, and DIANA-microT and enrichment analysis. Venn of 692 overlapping 1317 target genes (DIANA), 1328 target genes (miRDB) and 1338 target genes (TargetScan) (a). GO enrichment analysis, and KEGG (Kyoto Encyclopedia of Genes and Genomes) enrichment analysis revealed miR-19a-3p target genes correlated with multiple biological functions and pathways at Enrichr analysis tool (http://amp. pharm.mssm.edu/Enrichr/) (b-e). Cytoscape software was used to analyze core subnetworks in miR-19a-3p target genes (f)

sis and miR-19a-3p knockdown increased cell apoptosis $(\mathrm{p}<0.01)$ (Figure 2c).

\section{Predicting miR-19a-3p target genes}

miR-19a-3p target genes were estimated through 3 bioinformatics databases, including TargetScan, miRDB, and DIANA-microT. A total of 692 genes were overlapped in 3 bioinformatics databases (Figure 3a). The Enrichr analysis tool was used to analyze the correlated biological function and pathway of these overlapping target genes, the results of which revealed that these target genes are correlated with multiple biological function and pathway, such as negative regulation of cytoplasmic translation, protein kinase activity, and FoxO signaling pathway $(\mathrm{p}<0.05)$ (Figure 3. b-e). Besides, we analyzed the protein interaction network of these overlapping genes through the STRING analysis tool (Figure S1) and extract core subnetworks using the Cytoscape MCODE analysis tool. Finally, we obtained 5 significant core subnetworks and found that FBXO32 is a core gene in the core subnetworks $(\mathrm{p}<0.05)$ (Figure 3f).

\section{FBXO32 was a target gene of miR-19a-3p in multiple myeloma cells}

In CHAT, FBXO32 is also associated with sustaining proliferative signaling (Figure 4a). Then, we found that FBXO32 3'-UTR sequences matched the "seed sequence" of miR-19a-3p, which indicated FBXO32 is related to the regulation of MM (Figure 4b). Luciferase report system revealed that miR-19a-3p mimics significantly inhibits the activity of FBXO32-wt 3'UTR among H929 and U266 cells $(\mathrm{p}<0.05)$. miR-19a-3p inhibitors significantly promoted the activity of FBXO32-wt 3'UTR among H929 and U266 cells $(\mathrm{p}<0.01)$ (Figure 4. c, d). miR-19a-3p does not regulate the activity of FBXO32-mt 3'UTR among H929 and U266 cells (Figure 4. c, d). Besides, RT-qPCR assays revealed that FBXO32 is significantly down-regulated in patients with $\mathrm{MM}(\mathrm{p}<0.001)$ (Figure $4 \mathrm{e})$ and cells $(\mathrm{p}<0.05)$ (Figure 4f). miR-19a-3p mimics significantly inhibited FBXO32 mRNA expression $(\mathrm{p}<0.05$ ) (Figure 4g). miR-19a-3p inhibitors significantly promoted FBXO32 mRNA expression in U266 and H929 cells $(\mathrm{p}<0.001)$ (Figure 4h). 

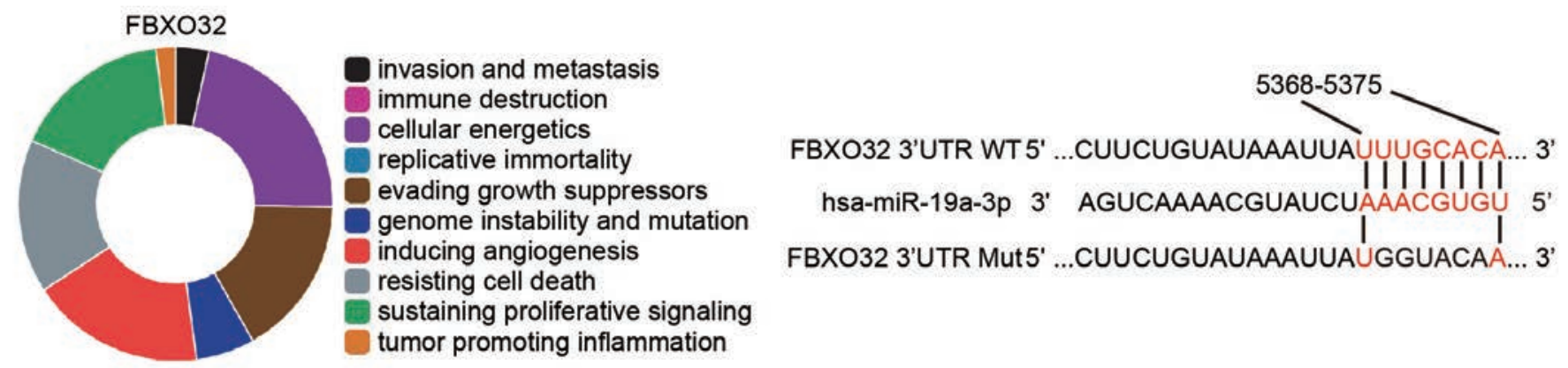

C

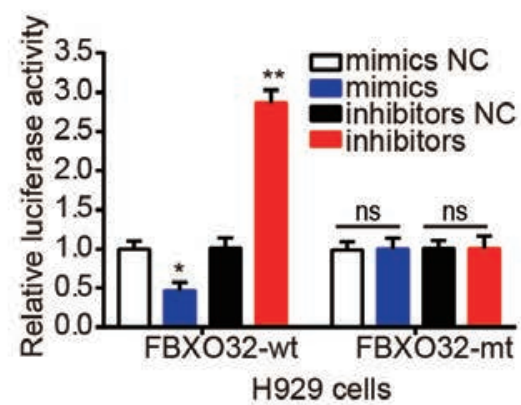

f

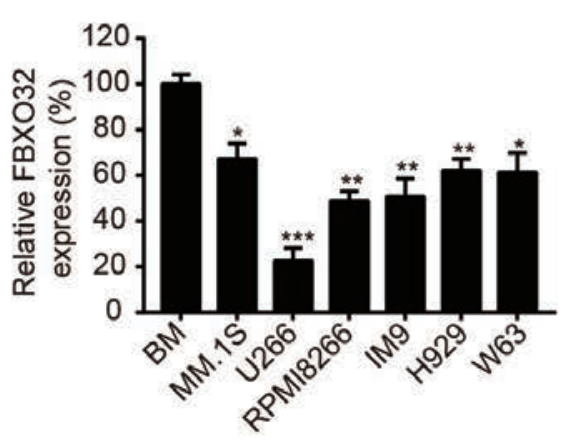

d

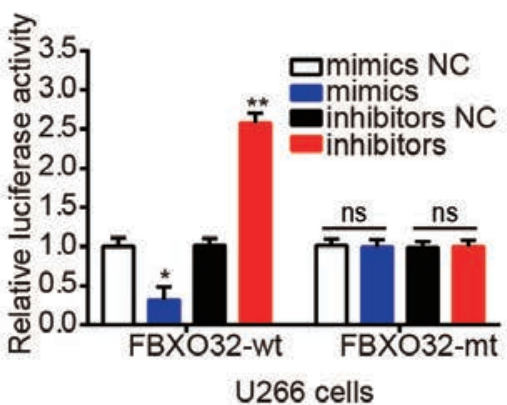

g

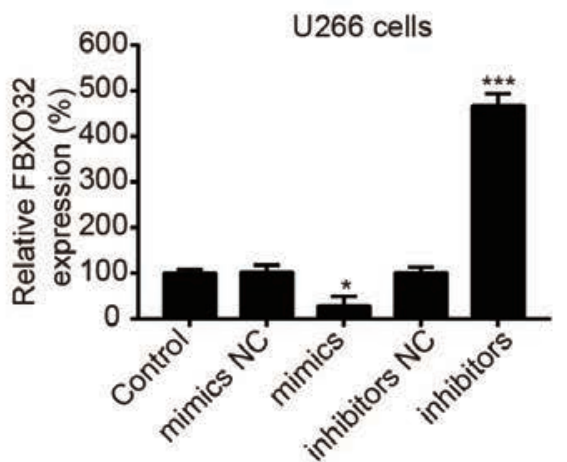

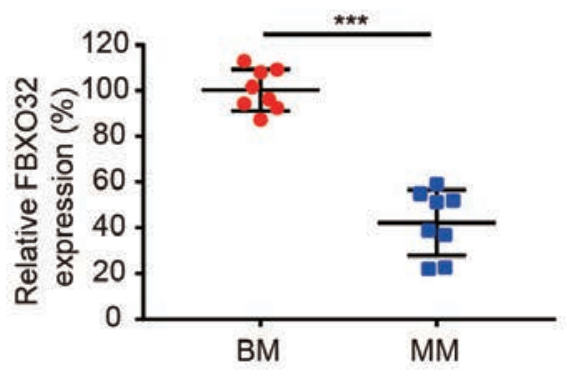

h

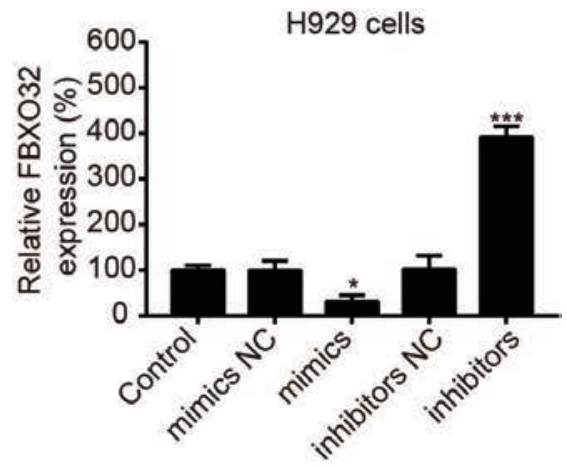

FIG. 4. a-h. FBXO32 acted as a direct target of miR-19a-3p in multiple myeloma (MM) cell lines. Association between FBXO32 and hallmarks of cancer from Cancer Hallmarks Analytics Tool (CHAT) (a). miR-19a-3p and its predicted binding sequences in the 3'-UTRs of FBXO32 Using TargetScan analysis tool (b). The dual-luciferase report system was used to detect the effect of miR-19a-3p on FBXO32 in H929 cells and U266 cells (c and d). Relative FBXO32 expression levels between the bone marrow (BM) and MM (e). Relative FBXO32 expression levels between BM and multiple myeloma cell lines (f). miR-19a-3p negatively regulated the expression of FBXO32 ( $g$ and $h$ ). ${ }^{*} p<0.05 ;{ }^{* *} p<0.01 ;{ }^{* * *} p<0.001$; each bar represented mean \pm standard deviation from 3 independent experiments

miR-19a-3p regulated cell viability and apoptosis through FBXO32 in multiple myeloma cells

FBXO32 was significantly overexpressed in U266 and H929 cells $(\mathrm{p}<0.01)$ (Figure 5a). CCK-8 assays revealed that FBXO32 significantly inhibits cell viability $(\mathrm{p}<0.05)$ (Figure $5 \mathrm{~b})$. Flow cytometry showed that FBOX32 significantly promotes cell apoptosis $(\mathrm{p}<0.01)$ (Figure 5. c, d). Furthermore, we found that FBXO32 significantly reverses the function of miR-19a-3p promoting cell viability $(\mathrm{p}<0.05)$ (Figure 5e). In addition, FBXO32 significantly reversed the function of miR-19a-3p inhibiting cell apoptosis $(\mathrm{p}<0.05)$ (Figure 5. f, g). WB revealed that miR-19a-3p signifi- cantly inhibits cleaved caspase 3 expression and increases $\mathrm{Bcl}-2$ expression, which was also reversed by $\mathrm{FBXO} 32$ overexpression $(\mathrm{p}<0.05)$ (Figure 5h).

\section{DISCUSSION}

MM remains an incurable hematological malignancy given the clinical use of chemotherapeutics, glucocorticoids, and novel treatments (25). Hence, it is essential to identify new biomarkers in tumorigenesis and progression, which contribute to realizing the pathogenesis and find novel treatment for MM. Recently, studies have demonstrated aberrant miRNA expression in carcinoma tis- 


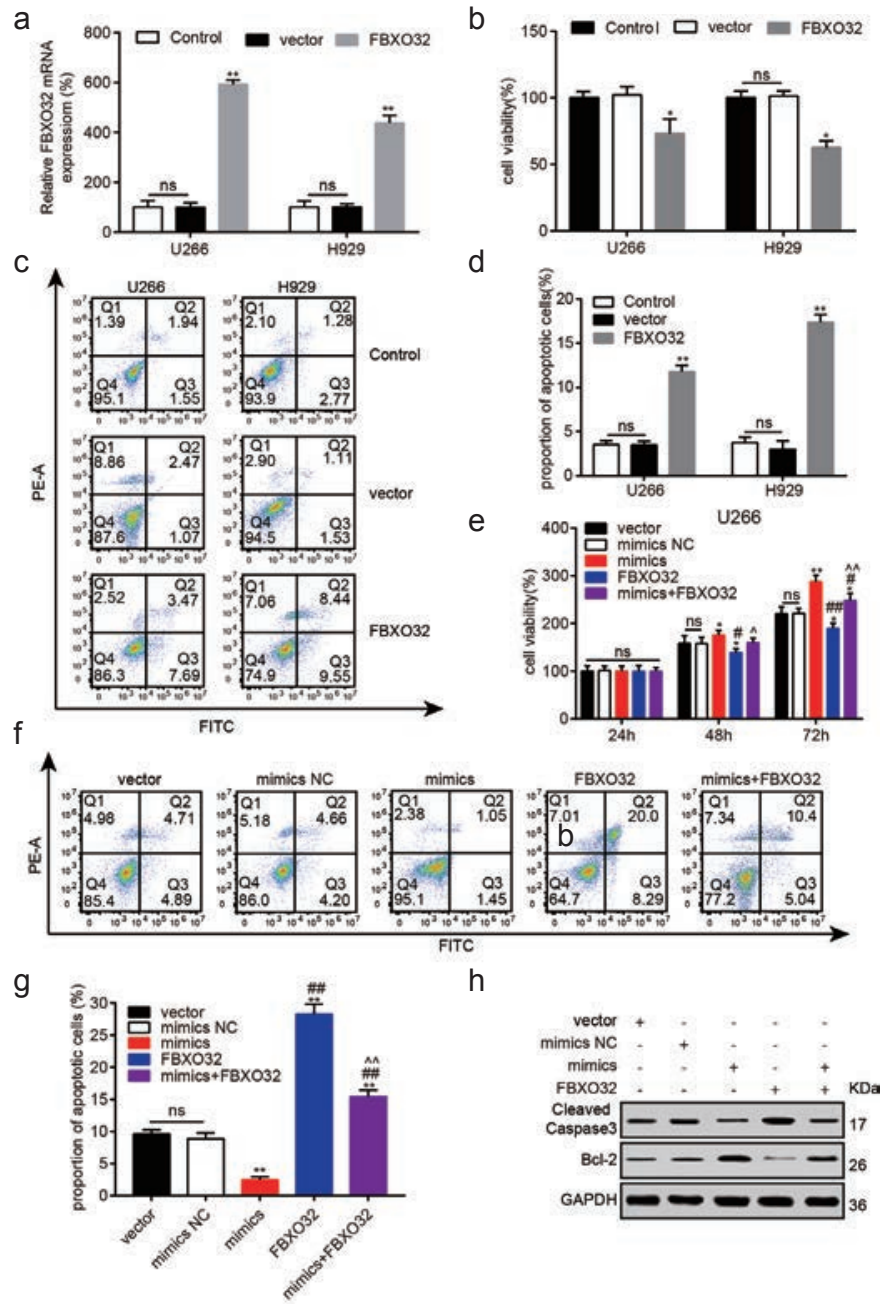

FIG. 5. a-h. miR-19a-3p regulates cell viability and cell apoptosis via FBXO32 in multiple myeloma (MM) cell lines. FBXO32 was overexpressed in U266 and H929 cells (a). Overexpressed FBXO32 significantly inhibited cell viability in U266 and H929 cells (b). Overexpressed FBXO32 significantly promoted cell apoptosis in U266 and H929 cells (c and d). FBXO32 rescued the effect of miR-19a-3p promoting cell viability (e). FBXO32 saved the result of miR-19a-3p inhibiting cell apoptosis ( $f$ and $g$ ). WB detected the expression of cleaved caspase 3 and BCL-2 (h). ${ }^{*} p<0.05$ vs vector group and mimics group, $\# p<0.05$ vs mimics group, $\wedge p<0.05$ vs FBXO3 group; each bar represented mean \pm standard deviation from 3 independent experiments.

sues and cells, which may indicate significant molecular and clinical implications of miRNA in tumorigenesis and progression (26).

Previous studies reported that miR-19a-3p was increased in many cancers (including MM, hepatocellular carcinoma, and breast cancer) $(5,27,28)$. Currently, our results presented that miR-19a-3p was overexpressed in MM cells and patients with MM (Figure 1. $\mathrm{b}, \mathrm{Cc})$. Interestingly, the significance level in patients with MM $(\mathrm{p}<0.001)$ was higher than in MM cells $(\mathrm{p}<0.01)$. Although the significance level does not account for the difference in expression, it is an interesting situation to consider. On the one hand, it may be caused by the lack of clinical samples. The miRNA expression lev- els of different individuals were significantly different. In contrast, these cell lines are derived from patients with MM, but screened. Cell lines are not equivalent to primary cells. Further studies revealed that miR-19a-3p suppressed cell apoptosis and promoted cell viability in U266 and H929 cells (Figure 2. a, c). These results showed that miR-19a-3p is an oncogene in MM cells. miRNAs alter gene expression by degrading or repressing target mRNAs. Subsequently, we predicted miR-19a-3p target genes in MM cells. Bioinformatics analysis ultimately extracted 5 core subnetworks (Figure 3f), which provides the research direction. Our previous studies screened these potential regulatory molecules. Eventually, we found that FBXO32 may be regulated by miR-19a-3p.

FBXO32 is a novel E3 ligase, which is one of the 4 subunits of the ligase complex of ubiquitin proteins (13). Recent evidence revealed the function of FBXO32 in tumorigenesis (29). FBXO32 was reported to be decreased in cancers, and FBXO32 induced apoptosis and increased cisplatin chemosensitivity (14-16, 29). Our results demonstrated that FBXO32 is down-regulated in MM cells and tissues (Figure 4. e, f). Besides, miR-19a-3p negatively regulated FBXO32 mRNA expression (Figure 4. g, h) in U266 and H929 cells. Luciferase assay verified that miR-19a-3p regulates gene expression by degrading FBXO32 mRNA (Figure 4. c, d).

Next, FBXO32 was overexpressed in U266 and H929 cells, and the results revealed that FBXO32 significantly suppressed cell viability and increased cell apoptosis (Figure 5. b, c). To verify that miR-19a-3p actually regulates a variety of biological functions by regulating FBXO32 expression in $\mathrm{MM}$, both mimics and $\mathrm{FBXO} 32$ overexpression plasmid were transfected in U266 cells, so as to detect cell viability, cell apoptosis, and apoptosis-associated protein expression. Our findings demonstrated that FBXO32 overexpression reverses the functions of miR-19a-3p increasing cell viability and suppressing apoptosis (Figure 5. e, f). In addition, FBXO32 overexpression (Figure 5h) abolishes the influences of miR-19a-3p suppressing cleaved caspase 3 and increasing BCL-2.

Based on the abovementioned results, we preliminarily revealed that miR-19a-3p directly regulates FBXO32. It would be significant to discover detailed mechanism in MM in the future. More studies are still necessary to confirm these findings, which will provide strong evidence supporting the function of miR-19a-3p and FBXO32 in MM occurrence and development.

Our current work confirmed the expression levels of miR-19a-3p in MM cells and patients with MM. miR-19a-3p significantly promoted cell viability and suppressed cell apoptosis. Moreover, FBXO32 was a target gene of miR-19a-3p. FBXO32 was down-regulated and significantly suppressed cell viability and promoted cell apoptosis in MM cells. Rescue assay further suggested that miR-19a-3p plays a role of oncogene by regulating FBXO32 expression in MM cells.

Ethics Committee Approval: Ethics committee approval was received for this study from the Ethics Committee of the Jinshan Hospital of Fudan University (JIEC-2018-04).

Patient Consent for Publication: Written informed consent was obtained from the all patients.

Author Contributions: Design - D.Z., Y.L.; Analysis and/or Interpretation - Y.Ma, Y.Meng.; Writing Manuscript - Y.L.; S.G. 
Data-sharing Statement: N/A.

Conflict of Interest: The authors have no conflicts of interest to declare.

Funding: This work was funded by the Youth Project of Jinshan District Health Committee (JSK-KTQN-2019-02).

\section{REFERENCES}

1. Kyle RA, Rajkumar SV. Multiple Myeloma. Blood 2008;111:2962-72. [Crossref]

2. Vicente-Dueñas C, Romero-Camarero I, García-Criado FJ, Cobaleda C, Sánchez-García I The Cellular Architecture of Multiple Myeloma. Cell Cycle 2012;11:3715-7. [Crossref]

3. Yang Y, Shi J, Tolomelli G, Xu H, Xia J, Wang H, et al. RAR $\alpha 2$ Expression Confers Myeloma Stem Cell Features. Blood 2013;122:1437-47. [Crossref]

4. Zhou W, Yang Y, Gu Z, Wang H, Xia J, Wu X, et al. ALDH1 Activity Identifies Tumor-Initiating Cells and Links to Chromosomal Instability Signatures in Multiple Myeloma. Leukemia. 2014;28:1155-8. [Crossref]

5. Zhang X, Chen Y, Zhao P, Zang L, Zhang Z, Wang X. MicroRNA-19a Functions as An Oncogene by Regulating PTEN/AKT/pAKT Pathway in Myeloma. Leuk Lymphoma. 2016;58:932-40. [Crossref]

6. Wang JH, Zhou WW, Cheng ST, Liu BX, Liu FR, Song JQ. Downregulation of Sprouty homolog 2 by microRNA-21 Inhibits Proliferation, Metastasis and Invasion, However Promotes the Apoptosis of Multiple Myeloma Cells. Mol Med Rep 2015;12:1810-6. [Crossref]

7. Lv H, Wu X, Ma G, Sun L, Meng J, Song X, et al. An Integrated Bioinformatical Analysis of miR-19a Target Genes in Multiple Myeloma. Exp Ther Med 2017;12:4711-20. [Crossref]

8. Rushworth SA, Murray MY, Barrera LN, Heasman S-A, Zaitseva L, MacEwan DJ. Understanding the Role of miRNA in Regulating NF-kB in Blood Cancer. Am J Cancer Res 2012;2:65-74

9. Shi Y, Jin Y. MicroRNA in cell differentiation and development. Sci China C Life Sci 2009;52:205-11. [Crossref]

10. Krol J, Loedige I, Filipowicz W. The Widespread Regulation of microRNA Biogenesis, Function and Decay. Nat Rev Genet 2010;11:597-610. [Crossref]

11. Hao M, Zang M, Wendlandt E, Xu Y, An G, Gong D, et al. Low Serum miR-19a Expression as a Novel Poor Prognostic Indicator in Multiple Myeloma. Int J Cancer 2015;136:183544. [Crossref]

12. Gomes MD, Lecker SH, Jagoe RT, Navon A, Goldberg AL. Atrogin-1, a Muscle-Specific F-Box Protein Highly Expressed During Muscle Atrophy. Proc Natl Acad Sci U S A 2001;98:14440-5. [Crossref]

13. Zhou H, Liu Y, Zhu R, Ding F, Wan Y, Li Y, et al. FBXO32 Suppresses Breast Cancer Tumorigenesis Through Targeting KLF4 to Proteasomal Degradation. Oncogene 2017;36:3312 21. [Crossref]

14. Chou JL, Su HY, Chen LY, Liao YP, Hartman-Frey C, Lai YH, et al. Promoter Hypermethylation of FBXO32, A Novel TGF-beta/SMAD4 Target Gene and Tumor Suppressor, Is Associated with Poor Prognosis in Human Ovarian Cancer. Lab Invest 2010;90:414-25.
[Crossref]

15. Guo W, Zhang M, Guo Y, Shen S, Guo X, Dong Z. FBXO32, A New TGF-beta/Smad Signaling Pathway Target Gene, Is Epigenetically Inactivated in Gastric Cardia Adenocarcinoma. Neoplasma 2015;62:646-57. [Crossref]

16. Wang C, Li X, Zhang J, Ge Z, Chen H, Hu J. EZH2 Contributes to 5-FU Resistance in Gastric Cancer by Epigenetically Suppressing FBXO32 Expression. Onco Targets Ther 2018;11:7853-64. [Crossref]

17. Guo W, Zhang M, Shen S, Guo Y, Kuang G, Yang Z, et al. Aberrant Methylation and Decreased Expression of the TGF- $\beta /$ Smad Target Gene FBXO32 in Esophageal Squamous Cell Carcinoma. Cancer 2014;120:2412-23. [Crossref]

18. Stitt TN, Drujan D, Clarke BA, Panaro F, Timofeyva Y, Kline WO, et al. The IGF-1/PI3K Akt Pathway Prevents Expression of Muscle Atrophy-Induced Ubiquitin Ligases by Inhibiting FOXO Transcription Factors. Mol Cell 20047;14:395-403. [Crossref]

19. Baker S, Ali I, Silins I, Pyysalo S, Guo Y, Högberg J, et al. Cancer Hallmarks Analytics Tool (CHAT): A Text Mining Approach to Organize and Evaluate Scientific Literature on Cancer. Bioinformatics 2017;33:3973-81. [Crossref]

20. Agarwal V, Bell GW, Nam JW, Bartel DP. Predicting Effective MicroRNA Target Sites in Mammalian mRNAs. Elife 2015;4:e05005. [Crossref]

21. Liu W, Wang X. Prediction of Functional Microrna Targets by Integrative Modeling of MicroRNA Binding and Target Expression Data. Genome Biol 2019;20:18. [Crossref]

22. Paraskevopoulou MD, Georgakilas G, Kostoulas N, Vlachos IS, Vergoulis T, Reczko M, et al. DIANA-microT Web Server v5.0: Service Integration into miRNA Functional Analysis Workflows. Nucleic Acids Res 2013;41:W169-73. [Crossref]

23. Kuleshov MV, Jones MR, Rouillard AD, Fernandez NF, Duan Q, Wang Z, et al. Enrichr: A Comprehensive Gene Set Enrichment Analysis Web Server 2016 Update. Nucleic Acids Res 2016;44:W90-7. [Crossref]

24. Szklarczyk D, Gable AL, Lyon D, Junge A, Wyder S, Huerta-Cepas J, et al. STRING v11: Protein-Protein Association Networks with Increased Coverage, Supporting Functional Discovery in Genome-Wide Experimental Datasets. Nucleic Acids Res 2019;47:D607-D13. [Crossref]

25. Harousseau JL, Moreau P. Autologous Hematopoietic Stem-Cell Transplantation for Multiple Myeloma. N Engl J Med 2009;360:2645-54. [Crossref]

26. Zhong $\mathrm{X}$, Coukos G, Zhang L. miRNAs in Human Cancer. Methods Mol Biol 2012;822:295-306. [Crossref]

27. Jiang XM, Yu XN, Liu TT, Zhu HR, Shi X, Bilegsaikhan E, et al. MicroRNA-19a-3p Promotes Tumor Metastasis and Chemoresistance through the PTEN/Akt Pathway in Hepatocellular Carcinoma. Biomed Pharmacother 2018;105:1147-54. [Crossref]

28. Yang J, Zhang Z, Chen C, Liu Y, Si Q, Chuang TH, et al. MicroRNA-19a-3p Inhibits Breast Cancer Progression and Metastasis by Inducing Macrophage Polarization Through Downregulated Expression of Fra-1 Proto-Oncogene. Oncogene 2014;33:3014-23. [Crossref]

29. Mei Z, Zhang D, Hu B, Wang J, Shen X, Xiao W. FBXO32 Targets c-Myc for Proteasomal Degradation and Inhibits c-Myc Activity. J Biol Chem 2015;290:16202-14. [Crossref] 


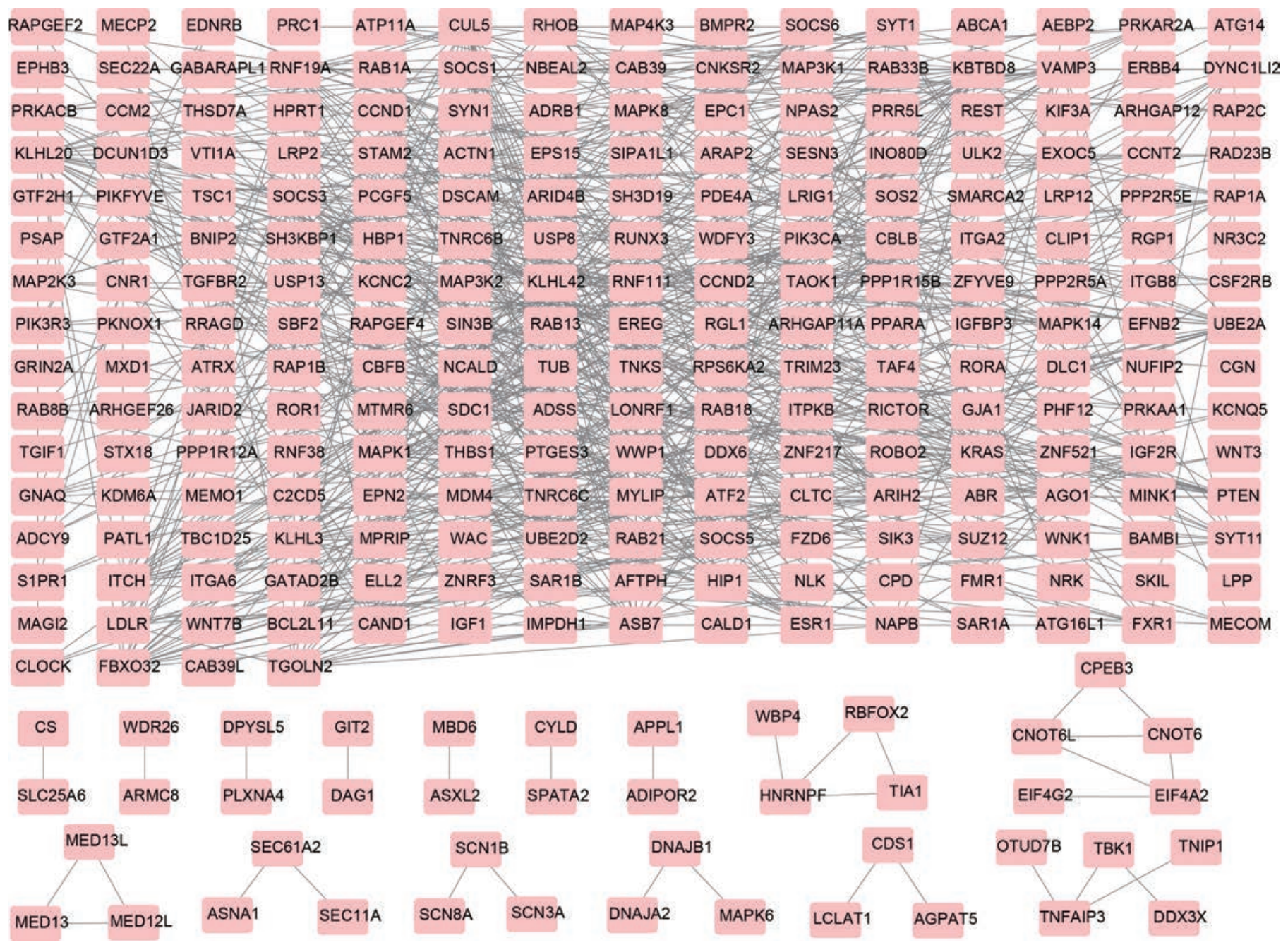

FIG. S1. Protein interaction network of miR-19a-3p target genes was analyzed at STRING analysis tools and visualized by Cytoscape software. 